\title{
Values of Radial Artery Provocation Tests at Different Doses of Ergonovine in the Diagnosis of Coronary Artery Spasm
}

\author{
Qing Zhang, ${ }^{1 *}$ MD, Bo Zhang, ${ }^{1 *}$ MD, Ye-fei Li, ${ }^{1}$ MD, Liang Chen, ${ }^{1}$ MD, Kou-long Zheng, ${ }^{1}$ MD, \\ Hui-he Lu, ${ }^{1} \mathrm{MD}$ and Zhen-qiang Sheng, ${ }^{1} \mathrm{MD}$
}

\begin{abstract}
Summary
The intracoronary drug provocation test has been the gold standard for diagnosis of coronary artery spasm (CAS); however, it has been identified with severe complications. In this study, we investigated the sensitivity, specificity, and safety of radial artery provocation test at different doses of ergonovine in the diagnosis of CAS. This study enrolled 57 patients, which were then divided into CAS group $(n=24)$ and control group $(n=33)$ after intracoronary ergonovine provocation test. All patients underwent radial artery provocation test at different doses of ergonovine. The predictive values of radial artery provocation test for the diagnosis of CAS were analyzed using receiver operator characteristic curve. In the radial artery provocation test at different doses of ergonovine, radial artery stenosis degree was all found to be significantly higher in the CAS group than in the control group (all $P<0.001$ ). In the control group, significant differences were noted in the radial artery stenosis degree between different doses of ergonovine (all $P<0.05$ ). In the CAS group, the radial artery stenosis degree was significantly higher in $160 \mu \mathrm{g}$ and $100 \mu \mathrm{g}$ of ergonovine than in $60 \mu \mathrm{g}$ of ergonovine (all $P<0.001$ ). The radial artery provocation test at $60 \mu \mathrm{g}$ and $100 \mu \mathrm{g}$ of ergonovine did not cause CAS, chest pain, and ECG ischemic changes. In the radial artery provocation test at $160 \mu \mathrm{g}$ of ergonovine, some patients had CAS, chest pain, and ECG ischemic changes. The specificity and sensitivity of radial artery provocation test were $90.91 \%$ and $50.00 \%$ at $60 \mu \mathrm{g}$ of ergonovine, $96.97 \%$ and $66.67 \%$ at $100 \mu \mathrm{g}$ of ergonovine, and $90.91 \%$ and $95.83 \%$ at $160 \mu \mathrm{g}$ of ergonovine for the diagnosis of CAS. As per our findings, we can conclude that the basic tension of radial artery increases in the CAS group. With the increase of ergonovine doses, its sensitivity and specificity improve, but its safety decreases. We will explore the most optimal dose of ergonovine in future studies.
\end{abstract}

(Int Heart J 2021; 62: 734-741)

Key words: Sensitivity, Specificity, Safety, Predictive value

$\mathrm{P}$ ast clinical data of 20 years have indicated that the coronary artery was normal or nearly normal in $50 \%$ of patients with stable angina and $10 \%-15 \%$ of patients with acute coronary syndrome. ${ }^{1)}$ Nowadays, ischemia with no obstructed coronary arteries (INOCA) has become a research hotspot, and a lot of evidence shows that coronary artery spasm (CAS) is one of the main causes of INOCA. ${ }^{2)}$ CAS refers to the transient spasm of the main coronary artery and its main branches, which leads to coronary artery occlusion and myocardial ischemia. ${ }^{3)}$ CAS can lead to angina pectoris, acute coronary syndrome, and even sudden cardiac death. ${ }^{4-8)}$ Therefore, accurate diagnosis of CAS is deemed of significant importance.

It is difficult to capture the direct evidence of CAS attack clinically as CAS has transient characteristics. The sensitivities of noninvasive tests are low for the diagnosis of CAS. Thus, the diagnosis of CAS usually depends on drug provocation test. The drugs used in the intracoronary provocation test include ergonovine and acetylcholine. At present, the intracoronary drug provocation test has become a gold standard for diagnosis of CAS. ${ }^{3)}$ However, the intracoronary drug provocation test, which is an invasive method, has been found to inevitably cause coronary intervention-related complications. If CAS is not relieved during the intracoronary drug provocation test, injecting nitroglycerin into the coronary artery is a must. Before the test, temporary pacemaker implantation is necessary for dealing with transient atrioventricular block, which may occur in intracoronary acetylcholine provocation test. The intracoronary drug provocation test may lead to severe complications such as ventricular tachycardia, ventricular fibrillation, bradycardia, cardiac shock, pericardial tamponade, acute myocardial infarction, and death. ${ }^{9}$

From the ${ }^{1}$ Department of Cardiology, The Second Affiliated Hospital of Nantong University, Nantong, China.

*These authors equally contributed to this study.

This study was supported by People's Livelihood Science and Technology Plan of Nantong Science and Technology Bureau (No. MSZ19183) and General Project B from Nantong Health Commission (No. MB2019013).

Address for correspondence: Zhen-qiang Sheng, MD, Department of Cardiology, The Second Affiliated Hospital of Nantong University, No. 6, Hai'er Xiang North Road, Chongchuan District, Nantong 226001, China. E-mail: 13962941081@163.com

Received for publication November 10, 2020. Revised and accepted February 15, 2021.

Released in advance online on J-STAGE July 17, 2021.

doi: $10.1536 /$ ihj. $20-735$

All rights reserved by the International Heart Journal Association. 
The exact mechanism of CAS has not been clearly determined. It has been reported that some factors such as smooth muscle hyperresponsiveness and vascular endothelial dysfunction are associated with CAS. ${ }^{10,11)}$ Under pathological conditions, blood vessel dysfunction is not only confined to a particular vessel, but it is also present in systemic vessels. ${ }^{12)}$ This makes it possible to evaluate coronary artery function by peripheral arteries. Compared with the intracoronary drug provocation test, provocation test of peripheral artery is more safe and simple, so it is a feasible method to use peripheral artery as a window to diagnose CAS.

The position of the radial artery is superficial, and both the radial artery and coronary artery are mediumsized arteries which are prone to spasm; thus, in this study, we explored the specificities and sensitivities of radial artery provocation test for the diagnosis of CAS at different doses of ergonovine.

\section{Methods}

All study methods were approved by the Ethics Committee of the Second Affiliated Hospital of Nantong University (2019KS079). All the subjects enrolled into the study gave written informed consent to participate.

Subjects and grouping: Patients who were admitted to our hospital due to repeated chest pain from January to December 2019 were examined. Inclusion criteria were as follows: (1) coronary stenosis $<50 \%$ determined via coronary angiography and (2) radial artery stenosis $<50 \%$ confirmed via radial angiography. Meanwhile, exclusion criteria were as follows: (1) age $>70$ years; (2) coagulation and/or hematopoietic diseases; (3) surgical history within 8 weeks; (4) severe cardiac insufficiency with left ventricular ejection fraction $(\mathrm{LVEF})<45 \%$; (5) history of myocardial infarction within 6 weeks; (6) patients with peripheral artery disease, proliferative retinopathy, or tumor; (7) patients with definite hypertrophic obstructive cardiomyopathy or valvular disease; (8) patients with a history of syncope or Adams-Stokes syndrome caused by bradyarrhythmias; and (9) patients with severe chronic obstructive pulmonary disease.

After excluding patients who were in line with the above exclusion criteria, 287 patients were examined for this analysis; however, 135 patients did not give consent to participate and thus, were excluded from the study. In the remaining 152 patients who provided consent, 94 had coronary stenosis $>50 \%$, and 1 patient had radial artery stenosis > 50\%; thus, they were terminated from the study. Finally, a total of 57 patients were enrolled in this study, who then all underwent intracoronary ergonovine provocation test. According to the diagnostic criteria of CAS as described in the guidelines for the diagnosis and treatment of coronary spasmodic angina pectoris made by Japanese Circulation Society (JCS), ${ }^{3)}$ the intracoronary ergonovine provocation test was positive in 24 patients and negative in 33 patients. The 24 patients were served as the CAS group and the 33 patients as the control group (Figure 1). All the 57 patients underwent radial artery provocation test at different doses of ergonovine.

General data: Within 24 hours after admission, all pa- tients received related examinations including blood pressure, routine blood test, fasting blood glucose, blood lipids, renal function, troponin I, and echocardiography.

Coronary angiography: The puncture of the left radial artery was performed by Seldinger's method, followed by placing a standard $6 \mathrm{~F}$ artery sheath. Heparin of $3000 \mathrm{U}$ was injected through the artery sheath, and then the left and right coronary angiography was performed by two independent observers using Judkins method. The diameter of the coronary artery was measured using QCA system.

Radial angiography: With the most strong brachial dance within the right elbow joint as a puncture point, puncture of the right brachial artery was performed via Seldinger's method, followed by placing an arterial indwelling needle $(220 \mathrm{G} / 1.10 \mathrm{~mm} \times 45 \mathrm{~mm}$, Becton Dickinson Infusion Therapy Systems Inc., Utah, USA) in the brachial artery at $30-45^{\circ}$ of left anterior oblique. The radial angiography was performed by two independent observers, and the diameter of the radial artery was measured using QCA system.

Intracoronary ergonovine provocation test: The intracoronary ergonovine provocation test was performed according to the 2013 JCS guidelines for the diagnosis and treatment of coronary spasmodic angina pectoris. ${ }^{3)}$ These patients must not take calcium antagonists and long-acting nitrate drugs within 48 hours and short-acting nitrate drugs within 6 hours. Before the intracoronary ergonovine provocation test, injecting intravenous or intracoronary nitroglycerin and other vasoactive drugs must be avoided. First, $60 \mu \mathrm{g}$ and $40 \mu \mathrm{g}$ of ergonovine (Chengdu Beite Pharmaceutical Co., Ltd; Chengdu, China) were respectively diluted in $5 \mathrm{~mL}$ of physiological saline and then were injected into the left and right coronary artery, respectively, within 3 minutes. One minute later, coronary angiography was performed. The interval of ergonovine injection between left and right coronary artery was 15 minutes. Positive criteria for the intracoronary ergonovine provocation test were as follows: (1) localized or diffuse CAS with a stenosis $>90 \%$ after injection of ergonovine and (2) chest pain attacks with or without ECG ischemic changes, followed by spontaneous remission within several minutes or disappearance after injection of nitroglycerin into the coronary artery.

Radial artery provocation test at different doses of ergonovine: Ergonovine $(60 \mu \mathrm{g}, 100 \mu \mathrm{g}$, and $160 \mu \mathrm{g})$ was respectively diluted in $5 \mathrm{~mL}$ of physiological saline and then injected into the radial artery within 3 minutes respectively. One minute later, the radial angiography was performed by two independent observers, and the diameter of the radial artery was measured using QCA system. The interval of ergonovine injection between each dose was 15 minutes. The inner diameters $\left(\mathrm{D}_{0}\right.$ and $\left.\mathrm{D}_{1}\right)$ of the radial artery before and after ergonovine injection were measured. The degree of the radial artery stenosis was calculated according to the formula $\left[\left(D_{0}-D_{1}\right) / D_{0}\right] \times 100 \%$ (Figures $2,3)$.

Safety for the ergonovine provocation test of radial artery: In all patients, blood pressure, heart rate, and ECG were monitored during the ergonovine provocation test of radial artery. Within 5 minutes after injecting the ergonovine, a small amount of contrast agent was injected 


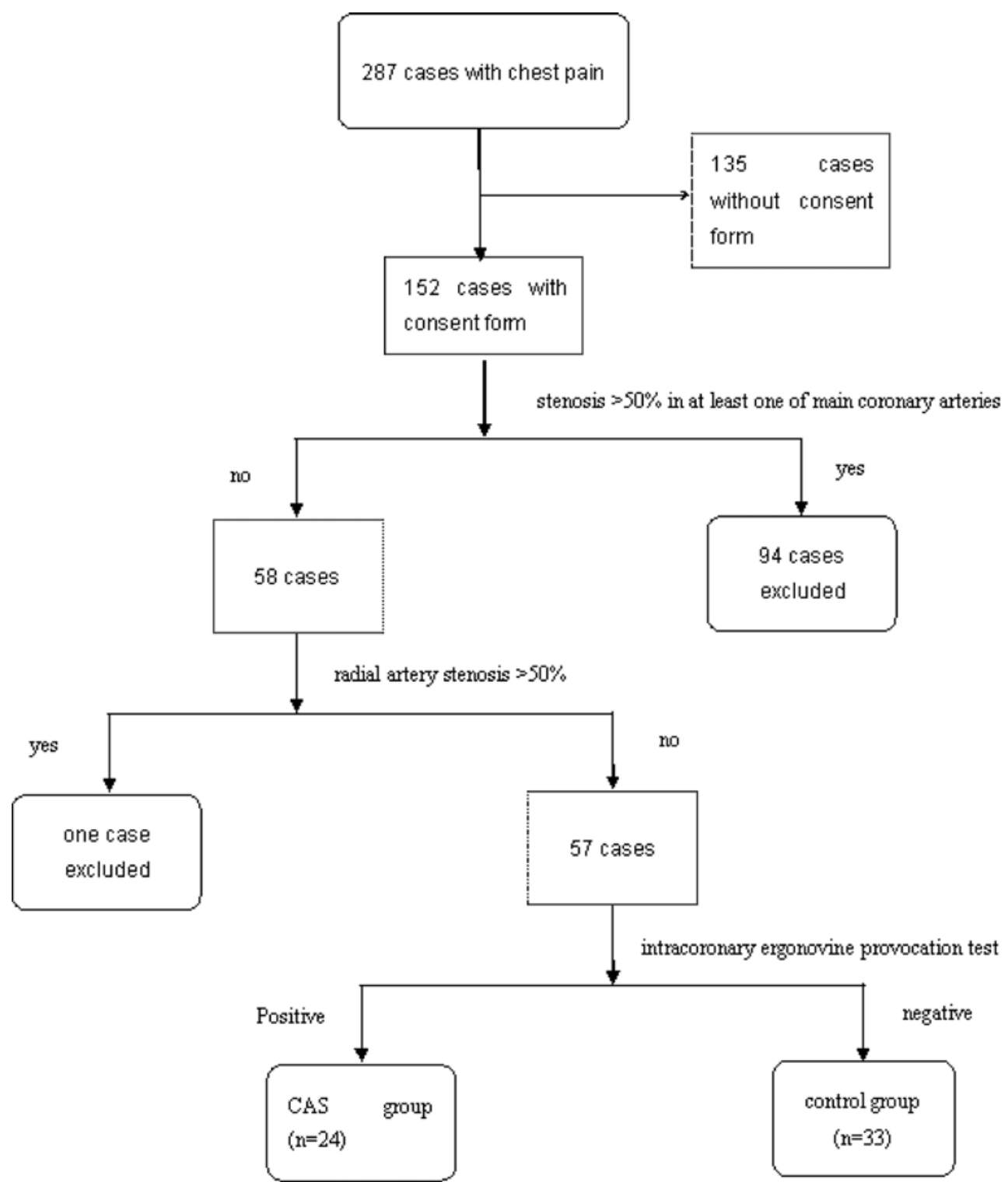

Figure 1. Flow chart of the cases included in this study. CAS indicates coronary artery spasm.

into the coronary artery every minute to observe the coronary artery. At the same time, we observed whether the chest pain and/or arrhythmias occurred, including sinus bradycardia, second or third degree atrioventricular block, ventricular tachycardia, or ventricular fibrillation. If CAS was not relieved consistently during the radial artery provocation test, nitroglycerin must be immediately injected into the coronary artery.

Statistical analysis: A power analysis was performed to determine the number of patients needed to distinguish significant differences between the two groups using PASS software, wherein the needed sample size was determined to be 28 cases per group ( $\alpha=0.15$ and $1-\beta=$ $0.9)$. The measurement data were expressed as mean \pm standard deviation. The enumeration data were expressed as percentage or frequency. The comparisons of data between the two groups were performed using independent sample $t$-test and $\chi^{2}$ test, respectively. Statistical analysis was performed using SPSS 23.0 software. The predictive values of radial artery provocation test for the diagnosis of CAS at different doses of ergonovine were analyzed by receiver operator characteristic (ROC) curves using MedCalc software. Statistical significance was established at $P$ $<0.05$.

\section{Results}

Comparisons of general data between the two groups: No significant differences were noted in terms of age, sex, diabetes prevalence, LVEF, body mass index, diastolic pressure, systolic pressure, fasting blood glucose, serum creatinine, blood urea nitrogen, triglyceride, total cholesterol, high density lipoprotein cholesterol, as well as taking aspirin, angiotensin-converting enzyme inhibitor/an- 


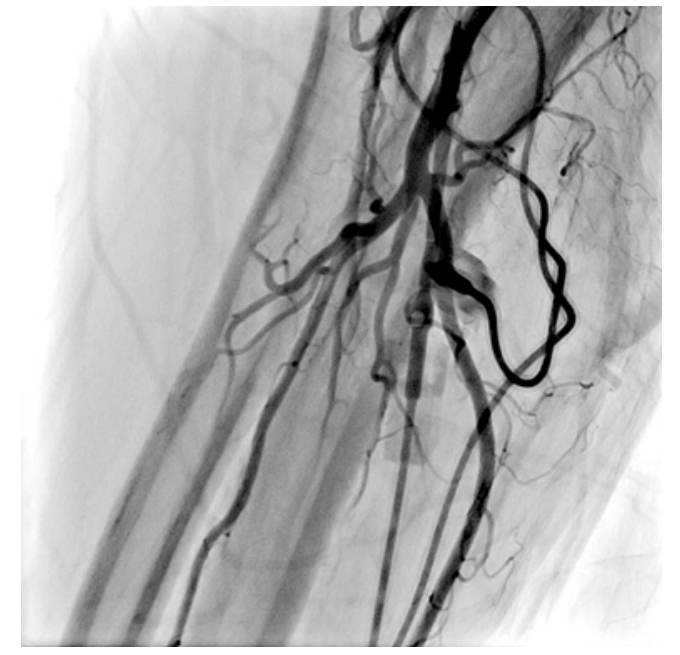

Figure 2. Radial artery provocation test indicated by radial angiography before injection of ergonovine into the radial artery.

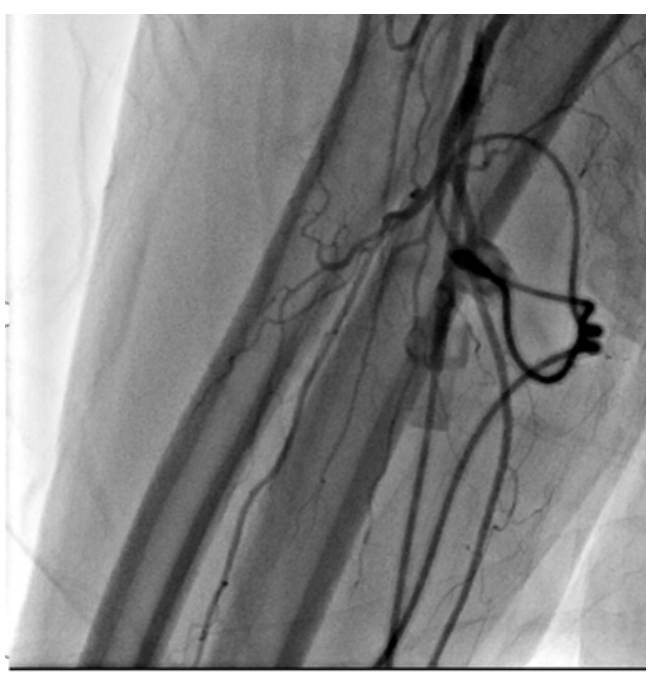

Figure 3. Radial artery provocation test indicated by radial angiography after injection of ergonovine into the radial artery.

giotensin receptor blocker, and other statins between the two groups (all $P>0.05$ ). Low-density lipoprotein cholesterol $(\mathrm{LDL}-\mathrm{C})(P=0.039)$ and smoking rate $(P=0.02)$ were significantly higher in the CAS group than in the control group (Table).

Radial artery provocation test at different doses of ergonovine: The spasm of radial artery was diffuse without localized spasm. The degree of the radial artery stenosis was all significantly higher in the CAS group than in the control group at $60 \mu \mathrm{g}(13.51 \% \pm 5.97 \%$ versus $2.27 \% \pm$ $0.77 \%), 100 \mu \mathrm{g}(39.54 \% \pm 5.62 \%$ versus $14.03 \% \pm$ $3.91 \%)$, and $160 \mu \mathrm{g}(49.5 \% \pm 7.32 \%$ versus $20.80 \% \pm$ $3.29 \%$ ) of ergonovine (all $P<0.001$ ) (Figure 4).

In the control group, the degree of the radial artery stenosis was significantly higher in the $100 \mu \mathrm{g}$ and $160 \mu \mathrm{g}$ of ergonovine than in the $60 \mu \mathrm{g}$ of ergonovine (all $P<$ 0.001 ) and in the $160 \mu \mathrm{g}$ of ergonovine than in the 100 $\mu \mathrm{g}$ of ergonovine $(P=0.041)$. In the CAS group, the degree of the radial artery stenosis was significantly higher in $160 \mu \mathrm{g}$ and $100 \mu \mathrm{g}$ of ergonovine than in $60 \mu \mathrm{g}$ of ergonovine (all $P<0.001$ ), but was not significantly different between $160 \mu \mathrm{g}$ and $100 \mu \mathrm{g}$ of ergonovine $(P=$ 0.051) (Figure 5).

Specificity and sensitivity of the radial artery provocation test for the diagnosis of CAS at different doses of ergonovine: The area under ROC curve of radial artery provocation test at $60 \mu \mathrm{g}$ of ergonovine for the diagnosis of CAS was 0.721 with $95 \%$ CI of $0.586-0.832, P=$ 0.001 , cut-off of $10 \%$, specificity of $90.91 \%$, and sensitivity of $50.00 \%$. The area under the ROC curve of radial artery provocation test at $100 \mu \mathrm{g}$ of ergonovine for the diagnosis of CAS was 0.864 with $95 \%$ CI of $0.747-0.940, P<$ 0.001 , cut-off of $35 \%$, specificity of $96.97 \%$, and sensitivity of $66.67 \%$. The area under the ROC curve of radial artery provocation test at $160 \mu \mathrm{g}$ of ergonovine for the diagnosis of CAS was 0.944 with $95 \%$ CI of $0.850-0.988, P<$ 0.001 , cut-off of $30 \%$, specificity of $90.91 \%$, and sensitivity of $95.83 \%$ (Figures 6-8).

Complications occurring in the ergonovine provocation tests of both coronary artery and radial artery: In the intracoronary ergonovine provocation test, one patient had premature ventricular contraction and one patient had paroxysmal atrial fibrillation in the control group, while, in the CAS group, one patient had paroxysmal atrial fibrillation and three patients had transient hypotension. All these complications disappeared after stopping ergonovine injection, and no fatal or severe complications such as persistent ventricular tachycardia, ventricular fibrillation, and myocardial infarction occurred.

The radial artery provocation test at $60 \mu \mathrm{g}, 100 \mu \mathrm{g}$, and $160 \mu \mathrm{g}$ of ergonovine did not cause severe complications, arrhythmia, and hypotension. In the radial artery provocation test at $60 \mu \mathrm{g}$ or $100 \mu \mathrm{g}$ of ergonovine, no CAS, chest pain, and ECG ischemic changes were noted to occur. In the radial artery provocation test at $160 \mu \mathrm{g}$ of ergonovine, one patient had CAS (90\% diffuse stenosis of the right coronary artery), chest pain, and ECG ischemic changes, one patient complained of chest pain alone, and one patient only had ECG ischemic changes in the CAS group, while in the control group, one patient had chest pain and ECG ischemic changes.

\section{Discussion}

Smoking and hyperlipidemia can induce oxidative stress in vascular wall and are the independent risk factors of CAS. ${ }^{13-16)}$ As per the findings of this study, it was found that the smoking rate and LDL-C were significantly higher in the CAS group than in the control group. Our results were similar to the results reported in the references. ${ }^{17,18)}$

The radial artery, $\alpha$-smooth muscle characteristic artery, has fine vessel diameter and is more sensitive to catecholamine in blood circulation, so placing guide wire, sheathing canal, and catheter can easily cause vasoconstriction, affecting the experimental results. In this study, the coronary angiography was performed by the puncture of the left radial artery, but the radial angiography and ra- 
Table. Comparisons of General Data Between the Two Groups

\begin{tabular}{lccl}
\hline \multicolumn{1}{c}{ Items } & $\begin{array}{c}\text { CAS group } \\
(n=24)\end{array}$ & $\begin{array}{c}\text { Control group } \\
(n=33)\end{array}$ & $P$ \\
\hline Age (years) & $56.8 \pm 1.0$ & $53.5 \pm 1.6$ & 0.110 \\
Sex (male/female) & $16 / 8$ & $21 / 12$ & 0.700 \\
Smoking $(\%)$ & 29.2 & 24.2 & 0.020 \\
Diabetes $(\%)$ & 12.5 & 15.2 & 0.200 \\
LVEF $(\%)$ & $63.5 \pm 1.1$ & $63.1 \pm 1.1$ & 0.229 \\
BMI $\left(\mathrm{kg} / \mathrm{m}^{2}\right)$ & $22.3 \pm 0.84$ & $24.0 \pm 0.7$ & 0.132 \\
Systolic pressure $(\mathrm{mmHg})$ & $133.0 \pm 5.1$ & $131.9 \pm 3.2$ & 0.8453 \\
Diastolic pressure $(\mathrm{mmHg})$ & $86.5 \pm 3.0$ & $84.4 \pm 2.3$ & 0.568 \\
Fasting blood glucose $(\mathrm{mM})$ & $5.80 \pm 0.20$ & $5.95 \pm 0.23$ & 0.640 \\
Serum creatinine $(\mu \mathrm{M})$ & $65.9 \pm 3.3$ & $66.5 \pm 2.8$ & 0.902 \\
Blood urea nitrogen $(\mathrm{mM})$ & $5.41 \pm 0.26$ & $5.23 \pm 0.22$ & 0.608 \\
Triglyceride $(\mathrm{mM})$ & $1.73 \pm 0.16$ & $1.55 \pm 0.15$ & 0.434 \\
Total cholesterol $(\mathrm{mM})$ & $4.59 \pm 0.16$ & $4.59 \pm 0.16$ & 0.993 \\
LDL-C $(\mathrm{mM})$ & $2.46 \pm 0.09$ & $2.16 \pm 0.10$ & 0.039 \\
HDL-C $(\mathrm{mM})$ & $1.25 \pm 0.28$ & $1.26 \pm 0.22$ & 0.8299 \\
ACEI/ARB $(\%)$ & 70.8 & 66.7 & 0.620 \\
Statins $(\%)$ & 50.0 & 48.6 & 0.910 \\
Aspirin $(\%)$ & 100 & 100 & 0.701 \\
\hline CAS indicas & &
\end{tabular}

CAS indicates coronary artery spasm; LVEF, left ventricular ejection fraction; BMI, body mass index; LDL-C, low-density lipoprotein cholesterol; HDL-C, high-density lipoprotein cholesterol; ACEI, angiotensin-converting enzyme inhibitor; and ARB, angiotensin receptor blocker.

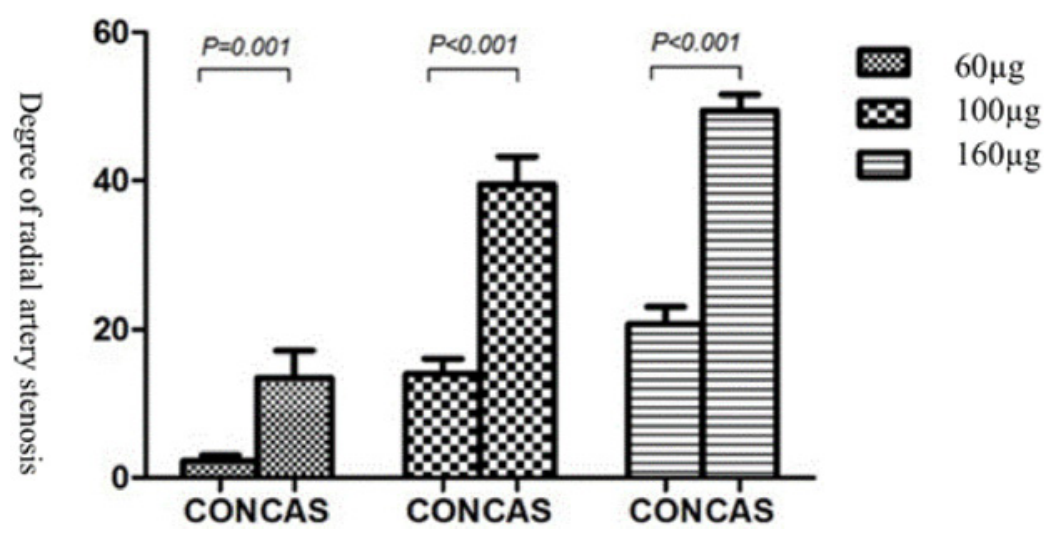

Figure 4. Comparisons of the radial artery stenosis degree between the two groups in the radial artery provocation test at different doses of ergonovine. CON indicates control group; and CAS, coronary artery spasm group.

dial provocation test were performed by the puncture of the right brachial artery followed by inserting an arterial indwelling needle. The puncture point was far from the right radial artery, which avoided the direct stimulation to the right radial artery; the diameter of the artery indwelling needle was fine, and the indwelling end was soft. These factors effectively avoided radial artery spasm induced by mechanical stimulation, ensuring the relative accuracy of the experimental results.

Both radial artery and coronary artery are prone to spasm and have many same independent predictors. The incidence of CAS-related events was determined to be higher in the patients with radial artery spasm than in the patients with non-radial artery spasm. ${ }^{19)}$ In this study, spasm of radial artery was diffuse without localized spasm, suggesting that the effect of ergonovine on radial artery was based on a universalistic elevation of basic tension in the radial artery. The radial artery stenosis degree was all significantly higher in the CAS group than in the control group at $60 \mu \mathrm{g}, 100 \mu \mathrm{g}$, and $160 \mu \mathrm{g}$ of ergonovine, suggesting that the blood vessel dysfunction was not only confined to the coronary artery, but also to the radial artery. In this study, the radial artery stenosis degree was significantly higher in $100 \mu \mathrm{g}$ and $160 \mu \mathrm{g}$ of ergonovine than in $60 \mu \mathrm{g}$ of ergonovine in both groups, suggesting that the radial artery spasm became more obvious with the increase of ergonovine. In the CAS group, the radial artery stenosis degree was found to be not significantly different between $100 \mu \mathrm{g}$ and $160 \mu \mathrm{g}$ of ergonovine $(P=0.051)$, which may be related to small sample size.

The areas under the ROC curves of radial artery 


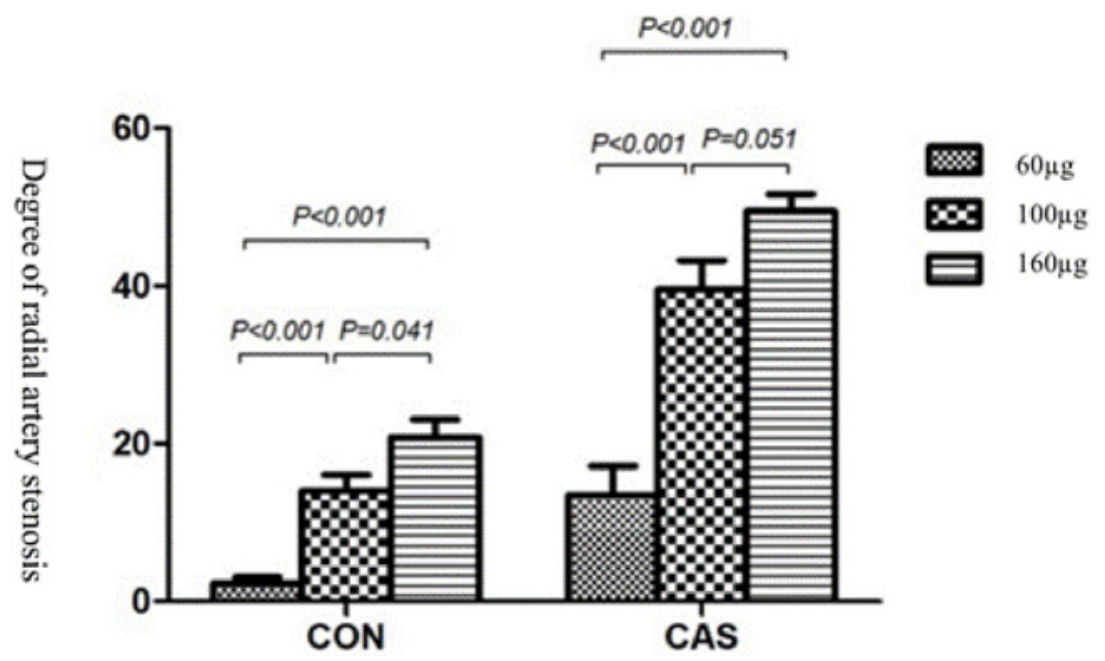

Figure 5. Comparisons of the radial artery stenosis degree between different doses of ergonovine in the radial artery provocation test in the control group and CAS group, respectively. CON indicates control group; and CAS, coronary artery spasm group.

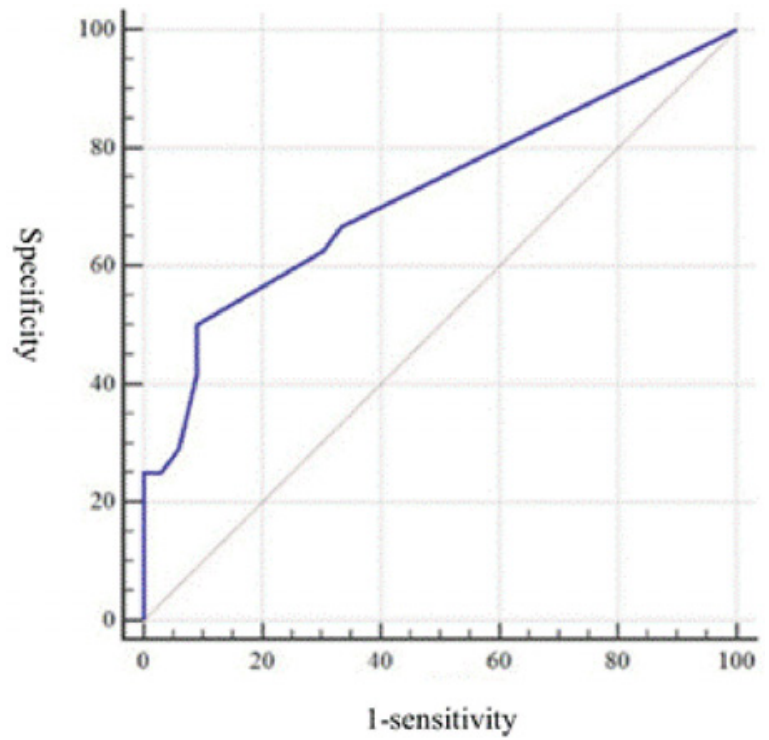

Figure 6. ROC curve of radial artery provocation test for the diagnosis of CAS at $60 \mu \mathrm{g}$ of ergonovine. ROC indicates receiver operator characteristic; and CAS, coronary artery spasm.

provocation tests at $60 \mu \mathrm{g}, 100 \mu \mathrm{g}$, and $160 \mu \mathrm{g}$ of ergonovine were $0.721,0.864$, and 0.944 (all $P<0.05$ ), respectively, suggesting that these radial artery provocation tests at $60 \mu \mathrm{g}, 100 \mu \mathrm{g}$, and $160 \mu \mathrm{g}$ of ergonovine all have values in the diagnosis of CAS. The area under ROC curve of radial artery provocation test at $60 \mu \mathrm{g}$ of ergonovine was the smallest, suggesting that $60 \mu \mathrm{g}$ of ergonovine was less effective in the provocation test of the radial artery, which led to low sensitivity and decreased diagnostic value. The area under ROC curve of radial artery provocation test at $160 \mu \mathrm{g}$ of ergonovine was the largest (0.944), with specificity of $90.91 \%$ and sensitivity of $95.83 \%$, suggesting that the accuracy of radial artery provocation test at $160 \mu \mathrm{g}$ of ergonovine was better for

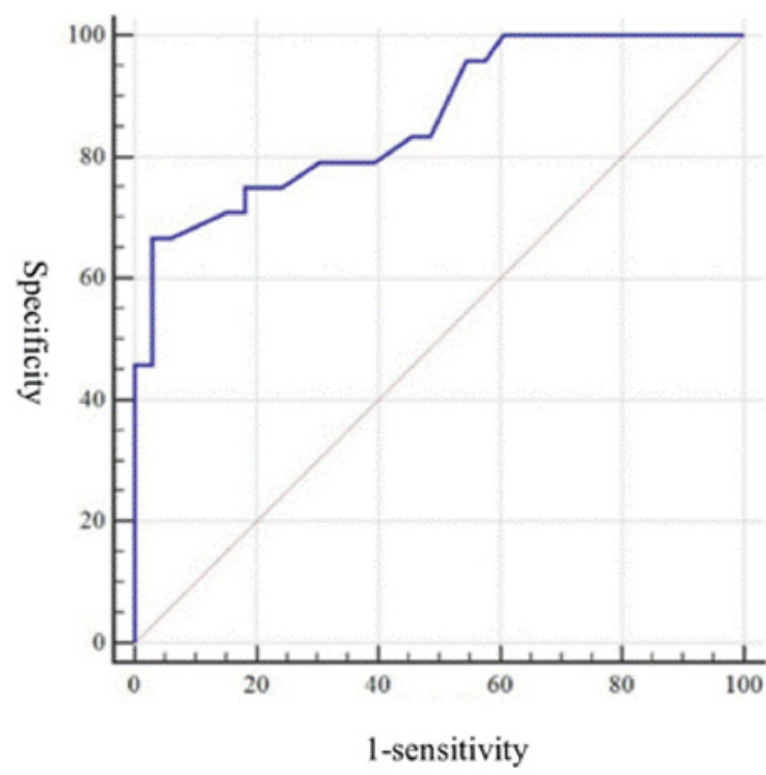

Figure 7. ROC curve of radial artery provocation test for the diagnosis of CAS at $100 \mu \mathrm{g}$ of ergonovine. ROC indicates receiver operator characteristic; and CAS, coronary artery spasm.

the diagnosis of CAS. The area under ROC curve of radial artery provocation test at $100 \mu \mathrm{g}$ of ergonovine was 0.864 , and its diagnostic value was between $60 \mu \mathrm{g}$ and $160 \mu \mathrm{g}$ of ergonovine.

For the intracoronary ergonovine provocation test, initially, $400 \mu \mathrm{g}$ of ergonovine was injected by peripheral vein, ${ }^{12)}$ which might have caused bilateral CAS at the same time and easily lead to serious complications due to intravenous systemic administration, ${ }^{20,21)}$ so the intravenous administration has been replaced by intracoronary administration. In this study, the radial artery provocation tests at $60 \mu \mathrm{g}$ and $100 \mu \mathrm{g}$ of ergonovine did not cause CAS, chest pain, and ECG ischemic change, suggesting that admini- 


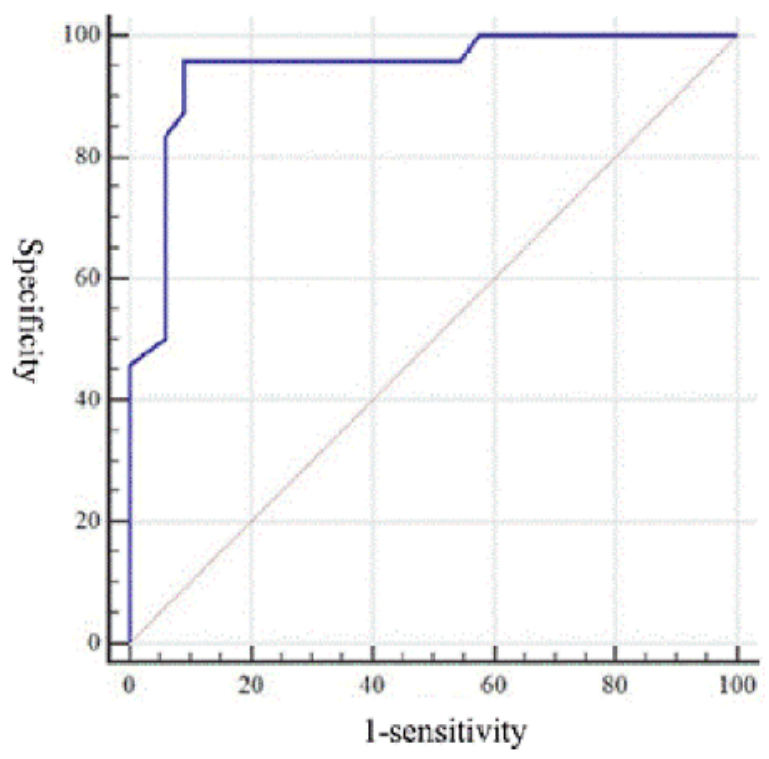

Figure 8. ROC curve of radial artery provocation test for the diagnosis of CAS at $160 \mu \mathrm{g}$ of ergonovine. ROC indicates receiver operator characteristic; and CAS, coronary artery spasm.

stration of ergonovine $(60 \mu \mathrm{g}$ or $100 \mu \mathrm{g})$ by brachial artery is safe. In this study, the administration by brachial artery also allowed the ergonovine to enter the bilateral coronary arteries at the same time, and $60 \mu \mathrm{g}$ and $100 \mu \mathrm{g}$ of ergonovine were more than the dose of ergonovine required by intracoronary ergonovine provocation test, but no complications occurred. This may be that the final concentration was not enough to induce CAS after the $60 \mu \mathrm{g}$ or $100 \mu \mathrm{g}$ ergonovine was diluted by systemic circulation and pulmonary circulation. In the radial artery provocation test at $160 \mu \mathrm{g}$ of ergonovine, one patient had CAS, chest pain and ECG ischemic changes, one patient had complained only of chest pain, and one patient only had ECG ischemic changes in the CAS group, suggesting that the safety of radial artery provocation test is related to the dose of ergonovine. The final concentration of $160 \mu \mathrm{g}$ ergonovine diluted by systemic circulation and pulmonary circulation was still able to induce CAS, so the safety of $160 \mu \mathrm{g}$ ergonovine was lower than that of $60 \mu \mathrm{g}$ and 100 $\mu \mathrm{g}$ of ergonovine.

The intracoronary ergonovine provocation test has high sensitivity and specificity, but the test is expensive, requires high technical level, and may cause complications, so it is only carried out in a few experienced heart centers. These limitations affect its wide application in clinical practice. The intracoronary ergonovine provocation test is invasive, so patients cannot receive this test repeatedly; thus, this test cannot be used for follow-up as well as efficacy evaluation and choice of drugs. Therefore, it is necessary to find a method which has high sensitivity, specificity, and safety and is easy to be carried out in clinical practice. Our results indicated that with the elevation of ergonovine, the specificity and sensitivity of radial artery provocation test increased. The specificity and sensitivity of $160 \mu \mathrm{g}$ ergonovine were better than that of 60 $\mu \mathrm{g}$ ergonovine or $100 \mu \mathrm{g}$ ergonovine, but $160 \mu \mathrm{g}$ er- gonovine might induce CAS, suggesting that with the increase of ergonovine, sensitivity increased but safety also decreased. The radial artery provocation test at $60 \mu \mathrm{g}$ of ergonovine did not induce CAS and myocardial ischemia, but its specificity and sensitivity were lower. The radial artery provocation test at $100 \mu \mathrm{g}$ of ergonovine also did not induce CAS and myocardial ischemia, and its sensitivity was higher than that of $60 \mu \mathrm{g}$ ergonovine. Whether there is a dose between $100 \mu \mathrm{g}$ and $160 \mu \mathrm{g}$ which can further improve the sensitivity and specificity under the premise of ensuring safety remains to be further explored.

In this study, there were some limitations. First, the sample size of this study was small. Second, the radial artery provocation test was also invasive and needed an $\mathrm{X}$ ray (QCA) for the judgment of the spasm. Therefore, it is necessary to increase the sample size and use ultrasound to observe the influences of ergonovine on the inner diameter of the radial artery instead of X-ray in future studies.

\section{Disclosure}

Conflicts of interest: None.

\section{References}

1. Bairey Merz CN, Pepine CJ, Walsh MN, et al. Ischemia and no obstructive coronary artery disease (INOCA): developing evidence-based therapies and research agenda for the next decade. Circulation 2017; 135: 1075-92.

2. Ford TJ, Rocchiccioli P, Good R, et al. Systemic microvascular dysfunction in microvascular and vasospastic angina. Eur Heart J 2018; 39: 4086-97.

3. JCS Joint Working Group. Guidelines for diagnosis and treatment of patients with vasospastic angina (coronary spastic angina) (JCS 2013). Circ J 2014; 78: 2779-801.

4. Prinzmetal M, Kennamer R, Merliss R, Wada T, Bor N. Angina pectoris. I. A variant form of angina pectoris; preliminary report. Am J Med 1959; 27: 375-88.

5. Ong P, Athanasiadis A, Borgulya G, Voehringer M, Sechtem U. 3-year follow-up of patients with coronary artery spasm as cause of acute coronary syndrome: the CASPAR (coronary artery spasm in patients with acute coronary syndrome) study follow-up. J Am Coll Cardiol 2011; 57: 147-52.

6. Yasue H, Kugiyama K. Coronary artery spasm: Japanese view. Coron Artery Dis 1990; 1: 668-74.

7. Sueda S, Fujimoto K, Sasaki Y, Habara H, Kohno H. Cardiogenic shock due to pulseless electrical activity arrest associated with severe coronary artery spasm. Intern Med 2018; 57: 28537.

8. Ferreiro RG, Roubín SR, Assi EA, Busto MC, Acuña JM, Juanatey JR. Noninvasive treatment of acute myocardial infarction. Clinical profile and predictors of poor prognosis. Rev Esp Cardiol (Engl Ed) 2015; 68: 343-5.

9. Matsumura M, Oshita C, Fujii Y, Ueda T, Teragawa H. Vasospastic angina diagnosed by the spasm provocation test with the combined use of the acetylcholine and ergonovine provocation tests. Intern Med 2019; 58: 2377-81.

10. Mehta PK, Thobani A, Vaccarino V. Coronary artery spasm, coronary reactivity, and their psychological context. Psychosom Med 2019; 81: 233-6.

11. Hubert A, Seitz A, Pereyra VM, Bekeredjian R, Sechtem U, Ong P. Coronary artery spasm: the interplay between endothelial dysfunction and vascular smooth muscle cell hyperreactivity. Eur Cardiol 2020; 15: e12. 
12. Bertrand ME, LaBlanche JM, Tilmant PY, et al. Frequency of provoked coronary arterial spasm in 1089 consecutive patients undergoing coronary arteriography. Circulation 1982; 65: 1299. 306.

13. Mashiba J, Koike G, Kamiunten H, Ikeda M, Sunagawa K. Vasospastic angina and microvascular angina are differentially influenced by PON1 A632G polymorphism in the Japanese. Circ J 2005; 69: 1466-71.

14. Figueras J, Domingo E, Ferreira I, Lidón RM, Garcia-Dorado D. Persistent angina pectoris, cardiac mortality and myocardial infarction during a 12 year follow-up in 273 variant angina patients without significant fixed coronary stenosis. Am J Cardiol 2012; 110: 1249-55

15. Mahemuti A, Abudureheman K, Schiele F, et al. Association between inflammatory markers, hemostatic markers, and traditional risk factors on coronary artery spasm in patients with normal coronary angiography. J Interv Cardiol 2014; 27: 29-35.

16. Ito T, Fujita H, Tani T, Sugiura T, Ohte N. Increased circulating malondialdehyde-modified low-density lipoprotein levels in patients with ergonovine-induced coronary artery spasm. Int J Car- diol 2015; 184: 475-80

17. Takagi Y, Yasuda S, Tsunoda R, et al. Clinical characteristics and long-term prognosis of vasospastic angina patients who survived out-of-hospital cardiac arrest: multicenter registry study of the Japanese coronary spasm association. Circ Arrhythm Electrophysiol 2011; 4: 295-302.

18. Yasue H, Mizuno Y, Harada E. Coronary artery spasm - Clinical features, pathogenesis and treatment. Proc Jpn Acad Ser B Phys Biol Sci 2019; 95: 53-66.

19. Varenne O, Jégou A, Cohen R, et al. Prevention of arterial spasm during percutaneous coronary interventions through radial artery: the SPASM study. Cathet Cardiovasc Interv 2006; 68: 231-5.

20. Ludmer PL, Selwyn AP, Shook TL, et al. Paradoxical vasoconstriction induced by acetylcholine in atherosclerotic coronary arteries. N Engl J Med 1986; 315: 1046-51.

21. Sueda S, Kohno H, Fukuda H, et al. Induction of coronary artery spasm by two pharmacological agents: comparison between intracoronary injection of acetylcholine and ergonovine. Coron Artery Dis 2003; 14: 451-7. 